\title{
Tissue coverage of paclitaxel and sirolimus eluting stents in long term follow-up: Optical coherence tomography study
}

\author{
Janusz Kochman, Arkadiusz Pietrasik, Adam Rdzanek, Anna Ścibisz, \\ Maciej Pawlak, Krzysztof J. Filipiak, Grzegorz Opolski \\ $1^{\text {st }}$ Chair and Department of Cardiology, Medical University of Warsaw, Poland
}

\begin{abstract}
Background: Implantation of drug eluting stents (DES) has become a standard treatment of patients undergoing percutaneous coronary intervention (PCI). Incomplete strut coverage is a potential risk factor for late stent thrombosis. Optical coherence tomography (OCT) enables in vivo identification of incomplete neointimal coverage.

Methods: Study included 62 patients after sirolimus eluting stents (SES) or paclitaxel eluting stents (PES) implantation. OCT examination was performed at least 24 months after the initial procedure (35.4 \pm 9.4 months). In cross-sectional still frames selected from each $1 \mathrm{~mm}$ of analyzed stents a total number of visible struts and number of struts with or without complete neointimal coverage was assessed. Measurements of neointimal coverage, presented as a mean thickness of tissue, were performed. Patients were followed up for 3 years and the frequency of major adverse cardiac events was recorded.

Results. In the analyzed 28 SES and 37 PES 9998 struts were identified. Complete neointimal coverage was observed in $83.5 \%$ and $79.2 \%$ of SES and PES struts respectively $(p=0.48)$. There was no difference in incidence of not covered or malapposed struts between SES and PES groups. Mean thickness of the tissue covering SES struts was $0.165 \pm 0.095 \mathrm{~mm}$, and $0.157 \pm 0.121 \mathrm{~mm}$ for PES. The mean neointimal thickness difference (SES vs. PES) was not statistically significant. In a 36 months follow-up 1 death was observed - potentially attributed to stent thrombosis.

Conclusions: A long term OCT follow-up after DES implantation shows high incidence of uncovered struts regardless of the stent type. Clinical significance of this finding remains questionable and requires further large scale trials. (Cardiol J 2013; 20, 3: 247-253)
\end{abstract}

Key words: PES, SES, restenosis, stent malapposition, stent expantion

\section{Introduction}

Implantation of drug eluting stents (DES) has become a standard method of treatment of patients with stable coronary artery disease (CAD) undergoing percutaneous coronary interventions
(PCI). Although DES reduced rates of restenosis compared with bare metal stents (BMS), late thrombosis, a life-threatening complication has emerged as a major concern. Incomplete strut coverage observed in follow-up period has been currently identified as a potential risk factor leading

Address for correspondence: Janusz Kochman, MD, $1^{\text {st }}$ Chair and Department of Cardiology, Medical University of Warsaw, ul. Banacha 1a, 02-097 Warszawa, Poland, tel: +48 2259919 58, fax: +48 22599 19 57, e-mail: jkochman@tlen.pl 
to the occurrence of late stent thrombosis (LST) $[1,2]$. In pathology studies incomplete neointimal coverage of stent struts was identified as powerful surrogate indicator of endothelialization and the most important morphometric predictor of LST [3].

Optical coherence tomography (OCT) is a novel diagnostic method that enables in vivo identification of incomplete coverage after stent implantation [4-6]. High OCT resolution allows for accurate evaluation of both the PCI effect and the vessel wall response to damage caused by the implanted stent. Additionally this method provides precise assessment of stent expansion and its apposition $[7,8]$.

Currently there is a limited number of published data regarding the comparison of struts coverage in paclitaxel (PES) and sirolimus (SES) eluting stents in a long term perspective. Hence, the purpose of present study was to assess the pattern of strut coverage in different types of DES in long term follow-up using OCT. Moreover, we sought to examine the relationship between strut coverage and clinical outcome.

\section{Methods}

The studied population comprised of patients with stable CAD who underwent PCI with implantation of PES (Taxus, Boston Scientific) or SES (Cyper, Cordis) between January 2003 and December 2005. Single-centre registry included 234 consecutive patients, 62 of them met the inclusion criteria and were involved in the study (Fig. 1).

The inclusion criteria were as follows:

- DES implantation in clinically and angiographically significant lesion of native vessel;

- At least 24 month since index PCI procedure;

- Stent diameter 2.5-3.5 mm.

Exclusion criteria:

- History of target vessel revascularization (TVR), myocardial infarction (MI) and stroke in a period between index PCI and planned OCT examination;

- Lack of capability to perform OCT measurement with proximal balloon occlusion (left main, proximal lesion $[<10 \mathrm{~mm}$ from native vessel ostium]);

- Implantation of DES in a chronic total occlusion of native artery;

- Chronic kidney disease with baseline GFR < $<30 \mathrm{~mL} / \mathrm{min} / 1.73 \mathrm{~m}^{2}$;

After OCT examination patients were prospectively followed-up for the period of 3 years. Frequency of major adverse events defined as all-cause

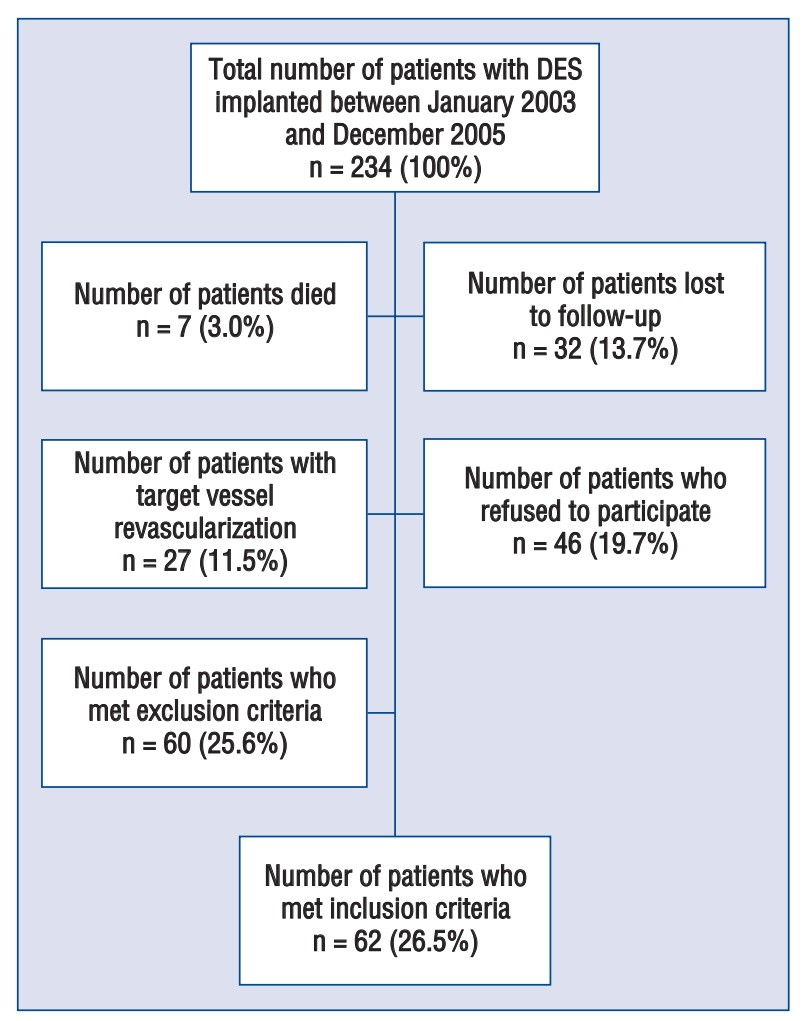

Figure 1. Patients enrollment flow chart; DES — drug eluting stents.

mortality, MI, stroke and repeat revascularization was registered.

The study was approved by the local research ethics committee and was conducted in accordance with Declaration of Helsinki.

\section{OCT data collection and analysis}

The OCT evaluation was performed in all patients included in the study using the M2 Cardiology Imaging System (LightLab Imagining Inc.) in accordance with manufacturer examination protocol. Low pressure occlusive over-the-wire balloon (Helios) was placed in the target vessel proximally to the lesion. An optic fibre probe (ImageWire, LightLab Imaging Inc.) was inserted through the balloon to the distal part of the vessel. After proximal balloon occlusion an automatic OCT pullback was performed at the speed of $2 \mathrm{~mm} / \mathrm{s}$ with a continuous Ringer lactate solution infusion.

The image analysis was performed based on the consensus of 2 experienced observers, blinded to the patients' clinical characteristics. Cross-sectional still frames were selected from each $1 \mathrm{~mm}$ of analyzed segment. Frames with major visual artefacts that prevented reliable assessment 


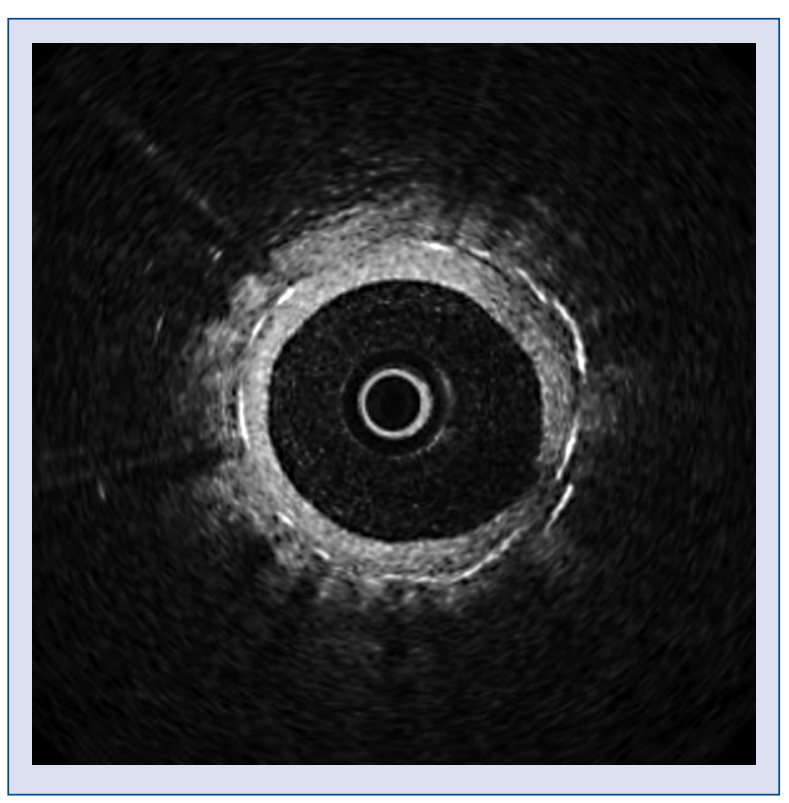

Figure 2. Strut with homogenous layer of tissue (covered).

of stent struts were excluded. In all remaining cross-sections the lumen and stent cross sectional area as well as the total number of visible struts and number of struts with and without coverage, were assessed. The measurements of the strut coverage, presented as a mean thickness of tissue, were performed on every visible stent strut ( 2 measurements per strut). Based on the OCT analysis 2 following stent strut coverage types were recognized: strut covered with homogenous layer of tissue (covered strut - Fig. 2) and strut without complete tissue coverage (uncovered strut — Fig. 3). Struts were considered to be "covered" if a layer of tissue was clearly visible on the strut surface. The OCT "uncovered" struts were identified when no evidence of tissue was visualized over the struts or if the strut was covered only partially.

Additionally cases of stent malapposition were identified. Malapposition was defined as a separation of the stent struts from the intimal surface of the arterial wall at a distance greater than the strut thickness including the polymer. Taking into consideration spatial resolution of the OCT $(20 \mu \mathrm{m})$, the malapposition was recognized when the strut distance from the vessel wall was greater than $160 \mu \mathrm{m}$ and $150 \mu \mathrm{m}$ for SES and PES, respectively.

\section{Statistical analysis}

The quantitative variables were presented as the mean values \pm standard deviation (SD). For categorical variables the relative and absolute

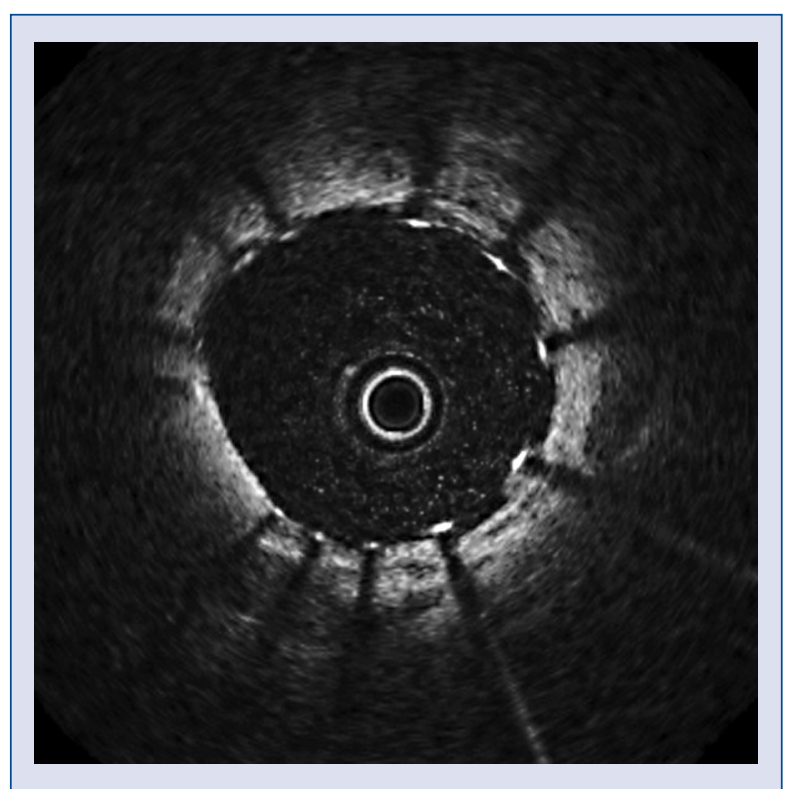

Figure 3. Strut without complete tissue coverage (uncovered).

frequencies are shown. The t-Student and Fischer tests were performed for subgroup comparisons. If the distribution was skewed a non-parametric test was used. A p value less than 0.05 was considered statistically significant. All analyses were performed using SAS software.

\section{Results}

The study population comprised of 62 patients with stable CAD who underwent PCI with DES implantation in de novo lesions. There were 45 $(72.6 \%)$ male, the mean age was $60.9 \pm 9.8$ year. In total there were 65 stents analyzed (28 SES and $37 \mathrm{PES})$. One patient in the SES group and 2 in the PES group received 2 stents in the target lesion. The mean follow-up time between the PCI and the OCT assessment was $35.4 \pm 9.4$ months.

Clinical characteristics and baseline angiographic data of the prespecified subgroups are presented in Tables 1 and 2 . There were no significant differences with regard to the risk factors of the cardiovascular disease and the angiographic profiles. The latter included target vessel, length and diameter of the stent, time from PCI to OCT assessment.

\section{OCT analysis}

In total, 1624 cross sections were obtained and 1552 (95.6\%) were included in the final analysis. There were 9998 struts identified, 4442 in the 
Table 1. Clinical characteristics of the study group.

\begin{tabular}{lcc}
\hline & SES ( $\mathbf{n} \mathbf{2 7}$ patients) & PES (n = 35 patients) \\
\hline Age [years] & 60.3 & 62.4 \\
Gender (male) & $16(59.3 \%)$ & $29(82.9 \%)$ \\
Previous myocardial infarction & $8(29.6 \%)$ & $10(28.6 \%)$ \\
Hypertension & $14(51.9 \%)$ & $21(60 \%)$ \\
Hyperlipidemia & $16(59.3 \%)$ & $29(82.9 \%)$ \\
Diabetes mellitus & $7(25.9 \%)$ & $11(31.4 \%)$ \\
Obesity & $6(22.2 \%)$ & $7(20 \%)$ \\
Smoker & $4(14.8 \%)$ & $5(14.3 \%)$ \\
Chronic kidney disease & $3(11.1 \%)$ & $3(8.6 \%)$ \\
Left ventricle ejection fraction $[\%]$ & $52 \pm 6$ & $55 \pm 7$ \\
Mean follow-up time & $37.2 \pm 11.7$ & $34.1 \pm 5.7$ \\
\hline
\end{tabular}

SES - sirolimus eluting stent; PES — paclitaxel eluting stent

Table 2. Baseline angiographic characteristics of the prespecified subgroups.

\begin{tabular}{lccc}
\hline & SES $(\mathbf{n}=\mathbf{2 8}$ stents) & PES (n = 37 stents) & P \\
\hline Target vessel & & & NS \\
Left anterior descending artery & $16(57.2 \%)$ & $20(54 \%)$ & NS \\
Circumflex artery & $6(21.4 \%)$ & $8(21.6 \%)$ & NS \\
Right coronary artery & $6(21.4 \%)$ & $9(24.4 \%)$ & NS \\
Lesion type: & & & \\
A & $4(14.3 \%)$ & $0(0 \%)$ & \\
B1 & $4(14.3 \%)$ & $6(16.2 \%)$ & NS \\
B2 & $9(32.1 \%)$ & $12(32.4 \%)$ & NS \\
C & $11(39.3 \%)$ & $3.12 \pm 0.38$ & NS \\
Mean stent diameter [mm] & $3.01 \pm 0.25$ & $24.62 \pm 5.8$ & NS \\
Mean stent length [mm] & $24.64 \pm 6.8$ & $4(10.8 \%)$ & NS \\
Direct stenting & $3(10.7 \%)$ & $31(83.8 \%)$ & $16.2 \pm 3.5$ \\
Post-dilatation & $23(82.1 \%)$ & $16.8 \pm 2.6$ & \\
Maximum inflation pressure & & & \\
\hline
\end{tabular}

SES - sirolimus eluting stent; PES — paclitaxel eluting stent

Table 3. Analysis of the optical coherence tomography (OCT) findings.

\begin{tabular}{|c|c|c|c|}
\hline & SES & PES & $\mathbf{P}$ \\
\hline Total number of cross sections & 684 & 940 & - \\
\hline Number of cross sections included in the analysis & 648 & 904 & - \\
\hline Number of analyzed struts & 4442 & 5556 & - \\
\hline Number of covered struts & $3711(83.5 \%)$ & $4402(79.2 \%)$ & 0.48 \\
\hline Number of uncovered struts & $731(16.5 \%)$ & $1154(20.8 \%)$ & 0.43 \\
\hline Mean tissue thickness covering the strut $[\mathrm{mm}]$ & $0.160 \pm 0.095$ & $0.157 \pm 0.121$ & 0.59 \\
\hline Number of malapposed struts & $87(1.9 \%)$ & $145(2.6 \%)$ & 0.68 \\
\hline Frequency of malapposition (\%) & $45(6.9 \%)$ & $56(6.2 \%)$ & 0.62 \\
\hline $\begin{array}{l}\text { Frequency of cross sections with the ratio } \\
\text { of covered struts/total struts }>30 \%\end{array}$ & $149(23.0 \%)$ & $273(30.2 \%)$ & 0.57 \\
\hline Neointimal hyperplasia area measured by OCT $\left[\mathrm{mm}^{2}\right]$ & $1.46 \pm 0.87$ & $1.59 \pm 1.34$ & 0.64 \\
\hline
\end{tabular}

SES - sirolimus eluting stent; PES — paclitaxel eluting stent 
SES group and 5556 in the PES group. Complete coverage was observed in $83.5 \%$ struts of the SES group and $79.2 \%$ of the PES group. The mean tissue thickness over the strut was $0.160 \pm 0.095$ $\mathrm{mm}$ and $0.157 \pm 0.121 \mathrm{~mm}$ in the SES and PES groups, respectively. In the SES group $2 \%$ and in the PES group $2.6 \%$ of struts met the malapposition criterion as defined above. There were $6.9 \%$ and $6.2 \%$ of cross sections showing malapposition in the SES and PES groups, respectively. Additionally we analyzed the number of cross sections with ratio of uncovered struts to total number of struts above 0.3 . There were no significant differences between the groups in terms of the OCT findings (Table 3 ).

The percentage of struts covered with the neointimal tissue of a thickness less than $150 \mu \mathrm{m}$, below intravascular ultrasound (IVUS) spatial resolution, was identified in $56.7 \%$ of the SES group and $61.8 \%$ of the PES group. The frequency distribution of neointimal thickness on stent struts is summarized in Figure 4.

\section{Clinical follow-up}

During the 36 months follow-up clinical data from $58(93.5 \%)$ patients was collected. There were 4 deaths (2 in SES group and 2 in PES group): 2 due to cancer, 1 due to stroke, 1 due to unknown reason (probable stent thrombosis according to ARC definition); 1 non-fatal MI of the non-culprit artery in the PES group; 1 patient required revascularization due to restenosis of the culprit vessel (SES group).

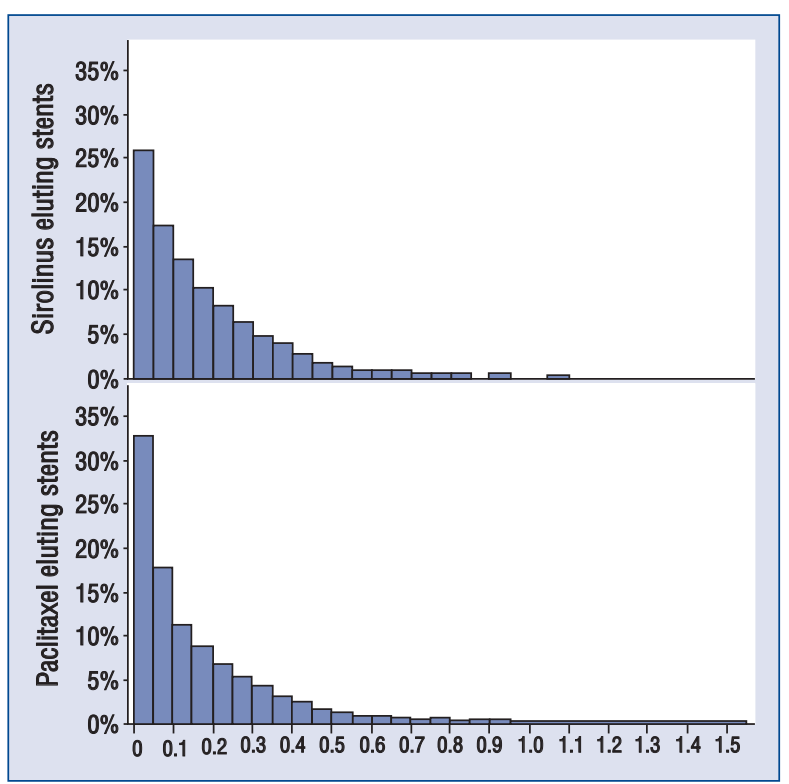

Figure 4. The frequency distribution of neointimal thickness on stent struts.

\section{Discussion}

The OCT a novel diagnostic method for the coronary artery imaging, introduced recently in to clinical practice, provides a new approach to a long term follow-up of patients after PCI procedures. Due to high spatial resolution OCT offers precise measurement of the neointimal thickness and distribution [9]. Additionally OCT enables assessment of tissue coverage of the each stent strut $[10,11]$. The struts are considered to be "OCT covered" if a layer of the tissue is clearly visible on surface of the struts. On the contrary, the "OCT uncovered" struts are recognized when no evidence of tissue could be visualized above the struts [12]. Stent apposition is another parameter which can be precisely assessed by OCT. The malappostion, defined as a separation of the stent struts from the intimal surface of the arterial wall at a distance greater than the strut thickness including the polymer, is caused mainly by positive remodeling and the absorption of the thrombus located between the stent and the vessel wall [13, 14]. This definition mainly depends on the strut thickness and may lead to ambiguity in the recognition of malapposition between different types of stents. The malapposition is often associated with uncovered struts and presence of the thrombus - the potential risk factors for the LST $[15,16]$. Although clinical implication of OCT derived stent assessment remains unknown, both neointimal coverage and the occurrence of malapposition seem to be important risk factors of stent thrombosis [17]. The issue of DES coverage was addressed in autopsy study published by Finn et al. [3]. In the multivariate analysis they identified the degree of the stent coverage as the significant predictor of LST in DES patients. The presence of $30 \%$ of uncovered struts per cross section accounted for nine fold increase in the risk of in-stent thrombus formation (odds ratio $=9.0 ; 95 \%$ CI 3.5-22). Additionally, in cases with thrombus present, a greater prevalence of incomplete strut apposition was found when compared to those without thrombus $(29 \%$ vs. $6 \%$; $p=0.02)$.

In our study we assessed the degree of strut coverage in DES in a long term follow-up using the OCT. In the studied population the high frequency of uncovered struts were observed, regardless of stent type. Late malapposition was a relatively rare phenomenon, equally distributed in both subgroups. Likewise, there were no differences in terms of number of uncovered struts and the neointimal thickness. The percentage of struts covered by the layer of tissue greater than $150 \mu \mathrm{m}$ 
were $56.7 \%$ and $61.8 \%$ in SES and PES group, respectively. This finding suggests that in majority of cases the IVUS, with its spatial resolution of more than $150 \mu \mathrm{m}$, might not be an adequate tool to assess the neointimal strut coverage.

In the study performed by Matsumoto et al. [18], 34 patients with total number of 57 sirolimus stents were assessed by both IVUS and OCT [18]. At 6 months only $16 \%$ of stents were identified to have all the struts fully covered with a visible layer of tissue. In the remaining $84 \%$ of stents, struts with no coverage were visualized by the OCT. Moreover, the percentage of struts covered with a layer of tissue undetectable by IVUS reached $64 \%$.

Healing pattern in different types of stents was also assessed by Chen et al. [19]. They compared the neointimal stent coverage in a group of 24 patients after SES and BMS implantation [19]. In the BMS patients in whom the OCT followup was completed between 5 to 10 months post stent implantation, OCT revealed the minimal and maximal neointimal thickness of 0.2 and $0.59 \mathrm{~mm}$, respectively. In the subgroup of patients at 23 to 93 months after BMS implantation these 2 parameters were comparable. This finding may suggest that the healing process is completed within first months post BMS implantation. In the SES group at the 6 to 12 months follow-up the minimal and maximal neointimal thickness was significantly smaller when compared to the BMS group $(0.04 \mathrm{~mm}$ and $0.12 \mathrm{~mm})$. In the BMS group $99.7 \%$ of struts were covered with tissue compared to $83 \%$ in the SES group. Moreover, malapposition defined as separation of the strut from the internal layer of the vessel wall of more than $200 \mu \mathrm{m}$, was significantly more frequent in SES group and accounted for $2 \%$ of cases.

Similar results were reported in the acute coronary syndrome (ACS) patients from the OCT substudy of HORIZONS AMI (Harmonizing Outcomes With Revascularization and Stents in Acute Myocardial Infarction) trial [20]. This study compared the safety and efficacy of primary angioplasty with PES or BMS implantation in patients with ST segment elevation MI. The OCT measurement was performed 13 months after PCI. In total 155 PES and 44 BMS were analyzed, which corresponded to over 40 thousand struts. In the BMS group $0.93 \%$ of struts were uncovered when compared to $4.79 \%$ in the PES group. The occurrence of strut malapposition was significantly higher in the PES group than in the BMS group ( $1.21 \%$ vs. $0.12 \%)$. In our study we found a relatively high incidence of uncovered struts despite of the long period from the implantation. Comparable results were reported by Kim et al. [21] who studied the ACS population treated with SES implantation. They also noticed significant difference in SES strut coverage between the ACS group and the stable CAD (17.3\% vs. $4.4 \%$; $\mathrm{p}<0.05$, respectively).

\section{Limitations of the study}

There are few limitations of our study that should be emphasized. First of all, this is a retrospective analysis of single-centre registry that included a limited number of patients after DES implantation. It is worth noticing that despite of relatively large number of struts included in the analysis, the limited number of stents, small size of the study population, retrospective character of the study and potential selection bias, do not allow for conclusive interpretation of the results. Further studies are needed to confirm the obtained outcomes.

Another constrain of our study derives from the limitation directly related to the OCT technique. The OCT resolution threshold does not allow for definite assessment of neointimal layer thinner than $20 \mu \mathrm{m}$. There is limited published data regarding direct comparison of the OCT images with the pathological findings. In the study by Prati et al. [22] 8 stents implanted in to the rabbit carotid artery was evaluated using the OCT technique. As reference method microscopic examination of the histopathology samples of the neointima was performed. OCT allowed proper identification of 97.4\% struts visible in pathological cross sections. In all examined cases OCT was as precise as microscopy in identification of both covered and uncovered struts. The degree of neointimal coverage correlated with the time from angioplasty and was equal to $0 \%, 12 \%, 41 \%$ and $97 \%$ in $2^{\text {nd }}, 7^{\text {th }}, 15^{\text {th }}$ and $28^{\text {th }}$ day of post PCI, respectively ( $<<0.001$ ). Furthermore, there were no differences in term of the tissue thickness over the strut as well as the mean cross-sectional area of the stent, as assessed by the corresponding methods. The minimal neointimal thickness measured in the pathological examination was $0.025 \mathrm{~mm}$ and was similar to the result obtained in the OCT $(0.024 \mathrm{~mm})$.

Results of the presented studies suggest that OCT may become a reliable reference method for long term evaluation of neointimal strut coverage and the PCI outcomes after stent implantation. However, stent endothelialization is a dynamic process and significant changes in tissue type and degree of strut coverage occur in a period of time. 
During the first months there is large proportion of fibrin in the newly formed tissue that cannot be reliably distinguished from the neointima in the OCT imaging. Hence, those struts that present with a tissue layer visualized in the OCT should be rather described as "OCT-covered", the term not equal to the real degree of the stent endothelialization.

\section{Conclusions}

The long term follow-up of stable coronary artery patients with complex coronary lesions treated with SES or PES angioplasty revealed a high occurrence of uncovered struts in the OCT examination. There were no significant differences in terms of degree of strut coverage and incidence of malapposition between the study groups. The constrains of the OCT and the lack of pathologic reference studies cast doubt on the clinical utility of the "OCT uncovered struts" as a potential surrogate endpoint of incomplete endothelialization and the healing process after stent implantation. The clinical implication of the OCT findings of the strut coverage requires verification in further studies.

\section{Conflict of interest: none declared}

\section{References}

1. Jimenez-Valero S, Moreno R, Sanchez-Recalde A. Very late drug-eluting stent thrombosis related to incomplete stent endothelialization: In-vivo demonstration by optical coherence tomography. J Invasive Cardiol, 2009; 21: 488-490.

2. Wenaweser P, Daemen J, Zwahlen M et al. Incidence and correlates of drug-eluting stent thrombosis in routine clinical practice. 4-year results from a large 2-institutional cohort study. J Am Coll Cardiol, 2008; 52: 1134-1140.

3. Finn AV, Joner M, Nakazawa G et al. Pathological correlates of late drug-eluting stent thrombosis strut coverage as a marker of endothelialization. Circulation, 2007; 115: 2435-2441.

4. Bouma BE, Tearney GJ, Yabushita $\mathrm{H}$ et al. Evaluation of intracoronary stenting by intravascular optical coherence tomography. Heart, 2003; 89: 317-320.

5. de Smet BJ, Zijlstra F. A look at drug eluting stents with optical coherence tomography. Eur Heart J, 2007; 28: 918-919.

6. Di Mario C, Barlis P. Optical coherence tomography: a new tool to detect tissue coverage in drug-eluting stents. J Am Coll Cardiol Cardiovasc Interv, 2008; 1: 174-145.

7. Barlis P, van Soest G, Serruys PW, Regar E. Intracoronary optical coherence tomography and the evaluation of stents. Expert Rev Med Devices, 2009; 6: 157-167.

8. Bezerra HG, Costa MA, Guagliumi G et al. Intracoronary optical coherence tomography: A comprehensive review clinical and research applications. J Am Coll Cardiol Cardiovasc Interv, 2009; 2: 1035-1046.

9. Murata A, Wallace-Bradley D, Tellez A et al. Accuracy of optical coherence tomography in the evaluation of neointimal coverage after stent implantation. J Am Coll Cardiol Cardiovasc Imaging, 2010; 3: 76-84.

10. Tanigawa J, Barlis P, Di Mario C. Intravascular optical coherence tomography: Optimisation of image acquisition and quantitative assessment of stent strut apposition. EuroIntervention, 2007; 3: 128-136.

11. Takano M, Yamamoto M, Inami S et al. Long term follow-up evaluation after sirolimus eluting stent implantation by optical coherence tomography. Do uncovered struts persist? J Am Coll Cardiol, 2008; 51: 968-969.

12. Barlis P, Dimopoulos K, Tanigawa J et al. Quantitative analysis of intracoronary optical coherence tomography measurements of stent strut apposition and tissue coverage. Int J Cardiol, 2010; 141: 151-156.

13. Prati F, Regar E, Mintz GS et al. Expert review document on methodology, terminology, and clinical applications of optical coherence tomography: Physical principles, methodology of image acquisition, and clinical application for assessment of coronary arteries and atherosclerosis. Eur Heart J, 2010; 31: 401-415.

14. Tanigawa J, Barlis P, Dimopoulos K et al. The influence of strut thickness and cell design on immediate apposition of drug-eluting stents assessed by optical coherence tomography. Int J Cardiol, 2009 15; 134: 180-188.

15. Bouki KP, Chatzopoulos DN, Katsafados MG et al. Late acquired stent malapposition detected by optical coherence tomography examination. Int J Cardiol, 2009; 137: e77-e78.

16. Savada T, Shite J, Shinke T el al. Very late throbosis of sirolimus eluting stent due to late malaposition: Serial observations with optical coherence tomography. J Cardiol, 2008; 52: 590-595.

17. Cook S, Wenaweser P, Togni M et al. Incomplete stent apposition and very late stent thrombosis after drug-eluting stent implantation. Circulation, 2007; 115: 2426-2434.

18. Matsumoto D, Shite J, Shinke T et al. Neointimal coverage of sirolimus-eluting stents at 6-month follow-up: Evaluated by optical coherence tomography. Eur Heart J, 2007; 28: 961-967.

19. Chen BX, Ma FY, Luo W et al. Neointimal coverage of bare-metal and sirolimus-eluting stents evaluated with optical coherence tomography. Heart, 2008; 94: 566-570.

20. OCT imaging suggests low rates of uncovered/malapposed struts following DES for AMI, www.theheart.org/article/921781.doi.

21. Kim JS, Fan C, Choi D et al. Different patterns of neointimal coverage between acute coronary syndrome and stable angina after various types of drug-eluting stents implantation; 9-month follow-up optical coherence tomography study. Int J Cardiol, 2011; 146: 341-346.

22. Prati F, Zimorano M, Stabile E et al. Does optical coherence tomography identify arterial healing after stenting? An in vivo comparison with histology, on a rabbit carotid model. Heart, 2008; 94: 217-221. 